\title{
Chip-Scale Photonics with Plasmonic Components
}

\author{
Harry A. Atwater, Luke A. Sweatlock, Jennifer A. Dionne, Robert J. Walters, \\ Julie S. Biteen, Sungjee Kim and Henri Lezec, Thomas J. Watson Laboratory of \\ Applied Physics, California Institute of Technology, MS 128-95, Pasadena, CA 91125, \\ haa@caltech.edu
}

Since the development of the light microscope in the 16th century, optical device size and performance has been limited by diffraction. Photonic devices of today are composed of dielectric materials with modest dielectric constants, and are much bigger than the smallest electronic devices for this reason. However subwavelength spatial confinement of light at dimensions down to less than $10 \%$ of the free-space wavelength is possible using plasmonic components. Ultimately it may be possible to employ plasmonic components to form the building blocks of a chip-based optical device technology that is scaleable to molecular dimensions, with potential imaging, spectroscopy and interconnection applications in computing, communication and chemical/biological detection.

In this talk we will describe recent opportunities presented by plasmonics for chip-scale integration of photonic and electronic devices, including i)design of metal-insulator-metal plasmon waveguides that optimize the trade-off between mode localization and propagation loss in the visible and nearinfrared ii) on-chip Si CMOS compatible light near-infrared light sources for coupling into plasmonic networks iii) plasmon-enhanced emission from quantum dots, and iv) opportunities for active plasmonic devices based on electro-optic and all-optical modulation of plasmon propagation. 